\title{
Dual Effects of Photo-Darkening and Photo-Bleaching in Ge-Se Films
}

István Csarnovics ${ }^{1,2}$, Muhamma Rizwa Latif ${ }^{2}$, Tyler Nichol ${ }^{1,2}$, Wan Kuang ${ }^{2}$, Maria Mitkova ${ }^{2}$, Miklós Veres ${ }^{3}$, Sándor Kökényesi ${ }^{4 *}$

1. Department of Experimental Physics, University of Debrecen, Debrecen 4026, Hungary

2. Department of Electrical and Computer Engineering, Boise State University, Boise, ID 83725-2075, USA

3. Institute of Solid State Physics and Optics, Wigner RCP, HAS, Budapest 1121, Hungary

4. Department of Electrical Engineering, University of Debrecen, Debrecen 4026, Hungary.

\begin{abstract}
Photo-induced changes are investigated in thermally evaporated $\mathrm{Ge}_{\mathrm{x}} \mathrm{Se}_{1-\mathrm{x}}(\mathrm{x}=0.28,0.33$ and 0.40$)$ films. Object of studies are fresh (as prepared), as well as annealed films. The studies indicated concentration dependence of the occurring effects for the studied compositions. Fast photo-darkening and slow photo-bleaching processes due to laser irradiation were detected in as-prepared films, while only photo-darkening effect was established for annealed samples. The origin of photo-darkening and photo-bleaching is explained based on the structural investigations carried out by Raman spectroscopy.
\end{abstract}

Key words: chalcogenides, photo-induced changes, photo-bleaching, photo-darkening, micro-Raman spectroscopy.

\section{Introduction}

Illumination with visible light causes a plurality of effects occurring in chalcogenide thin films like the well-known photo-induced structural transformation effects and related changes of optical parameters (absorption coefficient $\alpha$, refractive index $n$, etc.) [1], some peculiar effects like photo-plasticity or photo-fluidity, stimulated expansion or contraction and birefringence. Up to now these effects are rather ambiguously correlated with composition and experimental conditions [2-6], and the proposed mechanisms are sometimes contradictory.

During the last decade, a number of papers were devoted to the investigations of photo-induced effects for glasses from the Ge-Se systems [6-12]. It is reported [13, 14] that in contrast to As-based chalcogenides in which photo-darkening effect was observed, Ge-based chalcogenide glasses undergo photo-induced darkening and/or bleaching effect. A

Corresponding author: Sandor Kokenyesi, DSc., professor, research fields: materials science, photonics and plasmonics. E-mail: kiki@science.unideb.hu. few models have proposed that the intrinsic structural changes and/or photo-oxidation are responsible for photo-induced bleaching and for other structural modifications [15-17].

The aim of this work is to supply new data about the optical properties of the studied materials, and establish the compositional dependence of the photo-induced optical, structural and volume changes in thermally evaporated $\mathrm{Ge}_{x} \mathrm{Se}_{100-\mathrm{x}}$ thin films. Finally, we try to explain the fast photo-darkening and slow photo-bleaching effects from the view of optical and structural changes in them.

\section{Materials and Methods}

$\mathrm{Ge}_{\mathrm{x}} \mathrm{Se}_{100-\mathrm{x}}$ thin films $(\mathrm{x}=28,33$ and 40$)$ were prepared by thermal evaporation method in a Cressington 308R thermal evaporation system at $1 \times$ $10^{-6}$ mbar pressure, using a semi Knudsen cell crucible. The composition of the samples was measured with Energy Dispersive X-Ray Spectroscopy using Hitachi S-4300 system. The composition of the evaporated thin films were determined to be $\mathrm{Ge}_{28} \mathrm{Se}_{72}, \mathrm{Ge}_{33} \mathrm{Se}_{67}$ and $\mathrm{Ge}_{40} \mathrm{Se}_{60}$, slightly shifted towards increased Ge content 
from the original bulk glasses for the first two compositions due to the thermal evaporation conditions. The thickness $d$ of the samples was controlled by a calibrated evaporation process and was about $1000 \mathrm{~nm}$. Ambios XP-1 profilemeter was used to verify the sample thickness.

The optical transmission was detected by Agilent NIR-UV and Shimadzu UV-3600 spectrophotometers. The transmission spectra were recorded before and after irradiation. The kinetics of the transmission change under laser illumination was detected in situ by Newport and Thorlabs power meter setups. In fact, since the absorption edge and light sensitivity of the prepared samples depend on the films composition, its structure and Ge concentration [18], $\mathrm{Ge}_{28} \mathrm{Se}_{78}$ and $\mathrm{Ge}_{33} \mathrm{Se}_{67}$ samples were illuminated with laser setup operating at $533 \mathrm{~nm}$ with output power of $8.5 \mathrm{~mW}$, while the $\mathrm{Ge}_{40} \mathrm{Se}_{60}$ sample was irradiated with laser setup operating at $633 \mathrm{~nm}$ with an output power of $12 \mathrm{~mW}$. The samples were irradiated through a $2 \mathrm{~mm}$ aperture.

Refractive index, optical band gap, absorption coefficient and change of the absorption edge due to irradiation and annealing were calculated from the measured optical transmission spectra by Swanepoel method [19], while the thickness changes were measured by Ambios XP-1 profilemeter.

Photo-induced changes were investigated in annealed Ge-Se samples as well. Annealing was performed below the Ge-Se softening temperature $T_{g}$ (at $170{ }^{\circ} \mathrm{C}$ for films with composition $\mathrm{Ge}_{28} \mathrm{Se}_{72}, 300{ }^{\circ} \mathrm{C}$ for $\mathrm{Ge}_{33} \mathrm{Se}_{67}$ and $320{ }^{\circ} \mathrm{C}$ for $\mathrm{Ge}_{40} \mathrm{Se}_{60}$ ) for $2 \mathrm{~h}$ in vacuum.

The structural changes due to irradiation and annealing were measured by Raman spectroscopy using an Acton 275 spectrometer. For excitation, a p-polarized $514.5 \mathrm{~nm}$ line laser was focused into a circular spot of $1 \mathrm{~mm}$ diameter at a laser beam intensity of 25 W. Although the laser's wavelength is within the absorption edge of the films, no illumination-induced effects have been observed during and after several spectra measurements due to the very low beam intensity. The spectra were baseline-corrected, normalized and fitted with a set of Gaussian distributions to determine the peak parameters.

All data about PB (photo-bleaching) or PD (photo-darkening) results were measured at saturation of the effects on specific areas of the samples.

\section{Experimental Results and Discussion}

\subsection{Photo-Induced Effects in the As-Prepared Samples}

Fig. 1 represents the dependence of the transmission upon the wavelength for the studied films. The absorption spectra identify a clear dependence upon the film's composition with almost similar spectra for the $\mathrm{Ge}_{28} \mathrm{Se}_{72}$ and $\mathrm{Ge}_{33} \mathrm{Se}_{67}$ films and quite different spectrum for the $\mathrm{Ge}_{40} \mathrm{Se}_{60}$ film.

The as-deposited samples were irradiated with laser setups, as described in the experimental section. The measured spectra demonstrate occurrence of PD in the first seconds of irradiation which is followed by PB at subsequent light illumination, as presented in Fig. 2. The strong compositional dependence, noted in the absorption spectra measurement, is also represented in the photo-induced effects. The Se rich films react more explicitly to the laser light illumination with a tendency of decreasing of the effect appearance with growing of

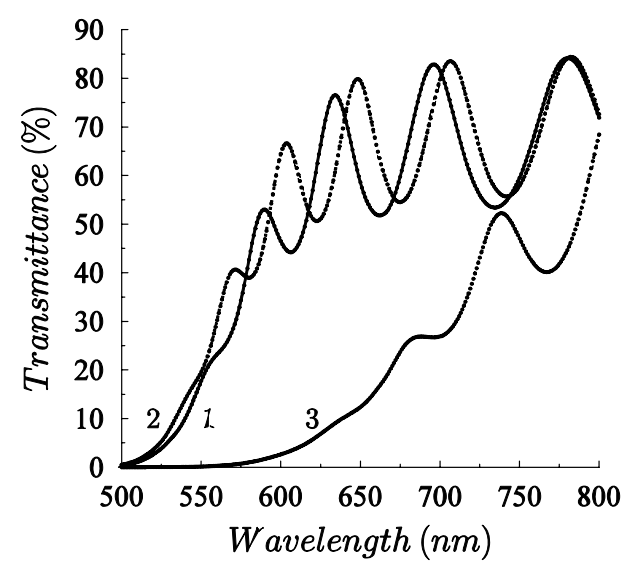

Fig. 1 Optical transmission spectra of the as-prepared Ge-Se samples: $1-\mathrm{Ge}_{22} \mathrm{Se}_{78}, 2-\mathrm{Ge}_{33} \mathrm{Se}_{67}$ and $3-\mathrm{Ge}_{40} \mathrm{Se}_{60}$. 
the Ge concentration in the films and with complete absence of PB for the samples containing $40 \% \mathrm{Ge}$ where barely noticeable photo-darkening effect exists. Note that the process reaches saturation after about 400 $\mathrm{s}$ for the $\mathrm{Ge}_{28} \mathrm{Se}_{72}$ and after about 1,800 $\mathrm{s}$ for the $\mathrm{Ge}_{33} \mathrm{Se}_{67}$ compositions at the given illumination conditions.

The optical transmission spectra of the samples were measured in their three states: Asdep (as-prepared), PD and PB state; and the corresponding optical parameters were calculated (Table 1).

The data show that: compared to the as-prepared state, the refractive index increases in the PD and decreases in the PB state, the value of the optical band gap is smaller in the PD and larger in the PB state. On the other hand, the absorption coefficient at the used laser wavelength is higher in the PD state and lower in the PB state. Increase of the thickness was detected in both PD and PB states for all the samples, and it was found to be prominent in the PD state. It was established that a more significant change in the optical parameters occurs for the $\mathrm{Ge}_{33} \mathrm{Se}_{67}$ sample, while the $\mathrm{Ge}_{28} \mathrm{Se}_{72}$ layer exhibited the largest change in the thickness during illumination.

The structural characteristics of the three studied compositions were measured by Raman spectroscopy. Characteristic peaks appeared at $202 \mathrm{~cm}^{-1}, 218 \mathrm{~cm}^{-1}$, and $255 \mathrm{~cm}^{-1}$, which correspond to the vibrations of the CS (corner-shared), ES (edge-shared) and $\mathrm{Se}-\mathrm{Se}$ (Se-Se chains) modes, respectively [20, 21]. For the Ge-richest sample, appearance of ETH (ethane) like structures at $168 \mathrm{~cm}^{-1}$ demonstrates a change in the structural organization.

From these spectra, the peak areas corresponding to each structural unit were determined for all three compositions.

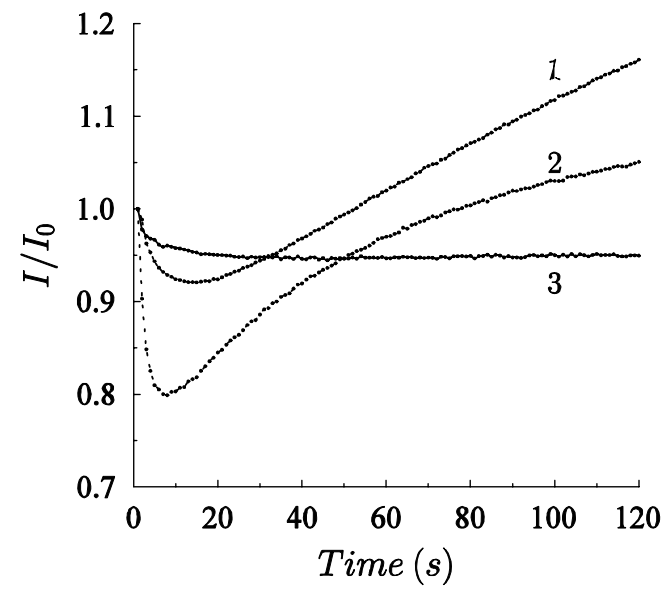

(a)

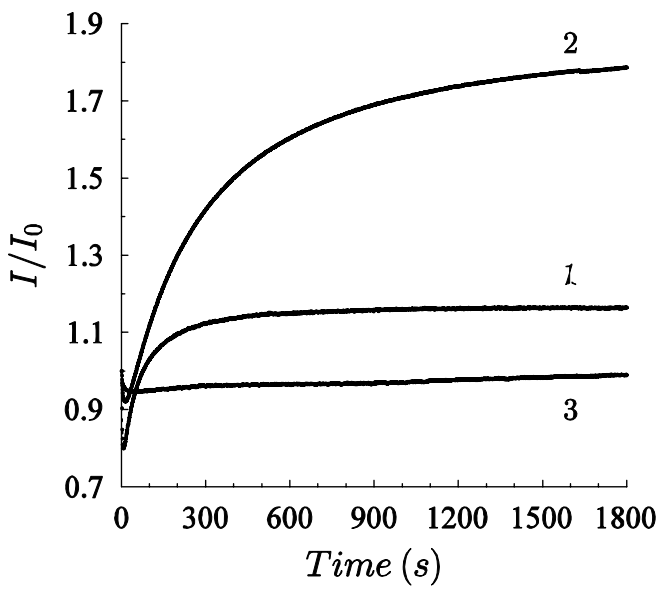

(b)

Fig. 2 Time dependence of the optical transmission of Ge-Se chalcogenide layers during laser irradiation, $1-\mathbf{G e}_{22} \mathrm{Se}_{78}, 2$ $\mathrm{Ge}_{33} \mathrm{Se}_{67}$ and $3-\mathrm{Ge}_{40} \mathrm{Se}_{60}$. (a): Photo-darkening process; (b): photo-darkening and photo-bleaching processes visible on a larger scale. The changes are normalized to absorption and illumination power.

Table 1 Calculated optical parameters (refractive index, absorption coefficient and optical band gap) and measured thickness of the irradiated Ge-Se samples at different stages of their illumination.

\begin{tabular}{llllllllll}
\hline & \multicolumn{3}{c}{$\mathrm{Ge}_{28} \mathrm{Se}_{72}$} & \multicolumn{3}{c}{$\mathrm{Ge}_{33} \mathrm{Se}_{67}$} & \multicolumn{3}{c}{$\mathrm{Ge}_{40} \mathrm{Se}_{60}$} \\
\cline { 2 - 10 } & Asdep & PD & PB & Asdep & PD & PB & Asdep & PD & PB \\
\hline$n$ & 2.52 & 2.53 & 2.50 & 2.48 & 2.49 & 2.46 & 2.92 & 2.93 & 2.93 \\
$d,(\mu \mathrm{m})$ & 1.10 & 1.13 & 1.12 & 1.20 & 1.23 & 1.22 & 1.15 & 1.16 & 1.16 \\
$\alpha, \mathrm{x} 10^{4}\left(\mathrm{~cm}^{-1}\right)$ & 2.26 & 2.45 & 2.11 & 1.91 & 1.97 & 1.62 & 1.55 & 1.59 & 1.58 \\
$E,(\mathrm{eV})$ & 1.99 & 1.98 & 2.01 & 2.21 & 2.20 & 2.22 & 1.78 & 1.78 & 1.78 \\
\hline
\end{tabular}




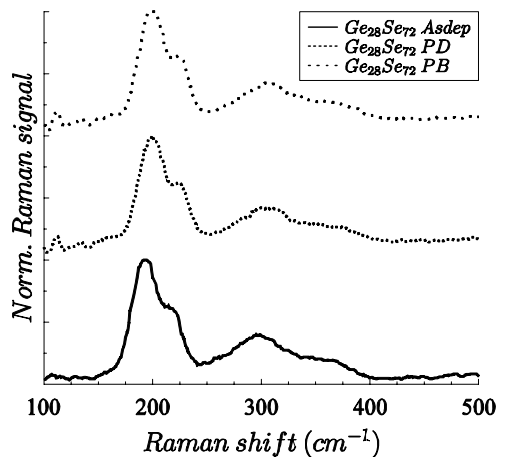

(1)

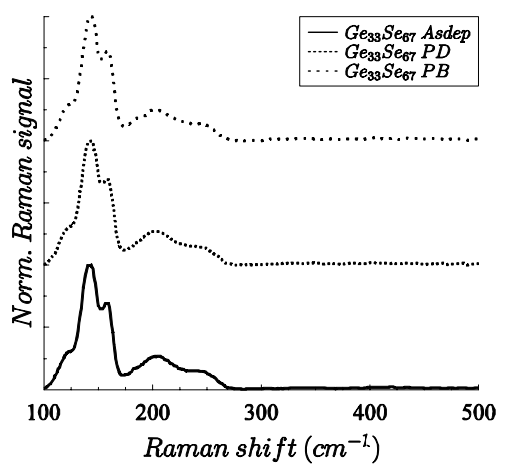

(2)

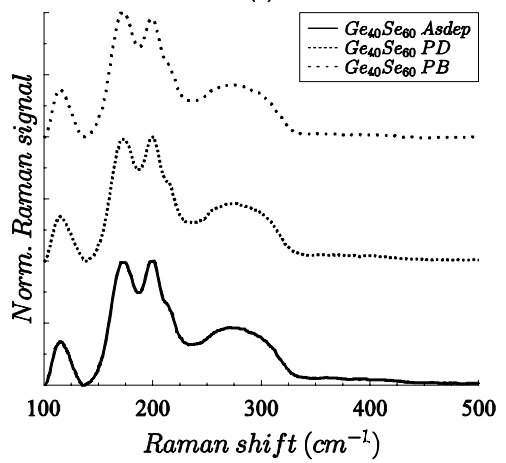

(3)

Fig. 3 Raman spectra of the as deposited and irradiated Ge-Se samples, (1) - $\mathrm{Ge}_{28} \mathrm{Se}_{72}$, (2) $-\mathrm{Ge}_{33} \mathrm{Se}_{67}$ and (3) $-\mathrm{Ge}_{40} \mathrm{Se}_{60}$.

The Raman spectra of the irradiated samples were measured in PD and PB states as well (Fig. 3). The area of the specific Raman peaks (Se-Se, ES, CS and ETH) is presented in Table 2. These data give some information about the structural changes taking place in the different states.

It is obvious from the data that: the area of the peak corresponding to homopolar bonds ( $\mathrm{Se}-\mathrm{Se}$ ) decreases in both the PD and PB states (probably the homopolar Se-Se bonds break and heteropolar Ge-Se bonds form, in agreement with the observations presented in [6]). The ES peak area decreases (the change is more remarkable in the PD state), the CS peak area increases (more significantly in the PD state). The ETH peak area decreases due to laser irradiation (relevant for $\mathrm{Ge}_{33} \mathrm{Se}_{67}$ and $\mathrm{Ge}_{40} \mathrm{Se}_{60}$ samples only).

\subsection{Photo-Induced Effects in Annealed Samples.}

The annealed samples were irradiated in the same way as the as-deposited ones. The time dependence of the optical transmission showed that laser irradiation causes only PD effect in the annealed samples (Fig. 4). No PB was observed for any of the Ge-Se compositions.

The optical parameters and thickness of the Ann (annealed) samples before and during the illumination (in the PD state) are presented in Table 3, together with the data of the Asdep samples for comparison.

From the data of the as-prepared and the annealed samples, it can be observed that annealing results in a decrease of the refractive index, thickness and absorption coefficient and an increase in the optical band gap.

The following effects were observed during the laser illumination of the annealed sample (in its PD state): the refractive index and absorption coefficient increase, together with the thickness, while the optical band gap decreases. The most significant change of the optical parameters was observed for the annealed $\mathrm{Ge}_{33} \mathrm{Se}_{67}$ film, while of the thickness change was more remarkable for the annealed $\mathrm{Ge}_{28} \mathrm{Se}_{72}$. It is worth mentioning that the change of the thickness due to irradiation in the annealed samples was found to be larger than in the as-prepared ones.

The comparison of the Raman spectra of the as-prepared annealed and irradiated annealed PD state is provided in Fig. 5, and the corresponding peak areas are given in Table 4.

Due to annealing the areal intensity of the Se-Se bonds, ETH and ES peak area decreases, while this for the CS increases. The irradiation of the annealed samples 
Table 2 Area of the major peaks in the Raman spectrum of the as deposited and irradiated Ge-Se samples, determined by fitting with Gaussians.

\begin{tabular}{llllllllll}
\hline & \multicolumn{3}{c}{$\mathrm{Ge}_{28} \mathrm{Se}_{72}$} & \multicolumn{3}{c}{$\mathrm{Ge}_{33} \mathrm{Se}_{67}$} & \multicolumn{3}{c}{$\mathrm{Ge}_{40} \mathrm{Se}_{60}$} \\
\cline { 2 - 10 } & Asdep & PD & PB & Asdep & PD & PB & Asdep & PD & PB \\
\hline Se-Se & 15.7 & 13.8 & 13.3 & 19.1 & 15.8 & 15.3 & 15.4 & 14.7 & 14.6 \\
ES & 7.2 & 6.5 & 6.8 & 5.1 & 4.9 & 5.0 & 3.4 & 3.2 & 3.3 \\
CS & 22.3 & 22.8 & 22.5 & 19.1 & 19.6 & 19.4 & 13.0 & 13.7 & 13.3 \\
ETH & - & - & - & 4.3 & 4.2 & 4.1 & 23.8 & 23.3 & 22.8 \\
\hline
\end{tabular}

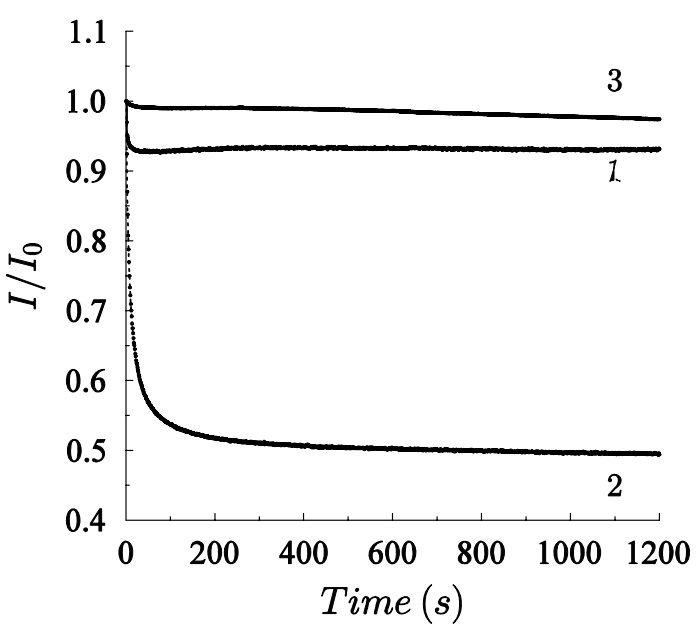

Fig. 4 Time dependence of the optical transmission for different annealed Ge-Se layers during laser irradiation at the proper laser wavelengths: $1-G_{22} \mathrm{Se}_{78}, 2-\mathrm{Ge}_{33} \mathrm{Se}_{67}$ and $3-\mathbf{G e}_{40} \mathrm{Se}_{6}$.

caused the following structural changes: Se-Se ETH, and CS peak area decreases and ES peak area increases.

\subsection{Discussion.}

One of the most important results of this work is the demonstration of transition of photo-darkening to photo-bleaching in thermally evaporated Ge-Se samples due to laser irradiation. The dual action of light was shown earlier in Ge-As-S [22, 23] and in Ge-As-Se [24] films. In essence, this shows the reversibility of the processes for which Elliott has created a unified model [25]. There are data missing how it relates to the compositional development of the samples and what is the nature of the effect.

The PD process starts after the laser irradiation is switched on, and saturates after 10-20 s. The change of the optical parameters (decreasing transmittance and blue shift of the absorption edge) can be explained by analyzing the structure of the material. As it was suggested earlier, the PD process can be connected with occurrence of electron-hole pairs, as a result of which, a non-uniform ("wrong") homopolar bond break and rearrangement to heteropolar bonds take place [25]. In fact, the occurrence of Se-Se bond breaking was proved by the Raman spectra. The Se-Se bond is the strongest in the studied system, however, light could cause some hybridization (mixing) of the lone-pairs and bond p-states at a top of the valence band [25]. This inevitably weakens the bond and because of this a preferable breaking of this bond is possible. Further effect of light illumination on the studied films is the breaking of some heteropolar bonds related to edge sharing structures which, due to their specific charge coupling, are weaker than those of

Table 3 Calculated optical parameters (refractive index, absorption coefficient and optical band gap) and measured thickness of the Asdep (as-prepared) and the annealed Ge-Se samples, before (Ann) and after laser irradiation (PD) of the latter.

\begin{tabular}{llllllllll}
\hline & \multicolumn{3}{c}{$\mathrm{Ge}_{28} \mathrm{Se}_{72}$} & \multicolumn{3}{c}{$\mathrm{Ge}_{33} \mathrm{Se}_{67}$} & \multicolumn{3}{c}{$\mathrm{Ge}_{40} \mathrm{Se}_{60}$} \\
\cline { 2 - 10 } & Asdep & Ann & PD & Asdep & Ann & PD & Asdep & Ann & PD \\
\hline$n$ & 2.52 & 2.50 & 2.51 & 2.48 & 2.47 & 2.48 & 2.92 & 2.91 & 2.91 \\
$d,(\mu \mathrm{m})$ & 1.10 & 1.08 & 1.16 & 1.20 & 1.18 & 1.25 & 1.15 & 1.12 & 1.15 \\
$\alpha, \mathrm{x} 10^{4}\left(\mathrm{~cm}^{-1}\right)$ & 2.26 & 2.07 & 2.37 & 1.91 & 1.22 & 1.56 & 1.55 & 1.62 & 1.70 \\
$E,(\mathrm{eV})$ & 1.99 & 2.02 & 2.00 & 2.21 & 2.24 & 2.22 & 1.78 & 1.79 & 1.79 \\
\hline
\end{tabular}




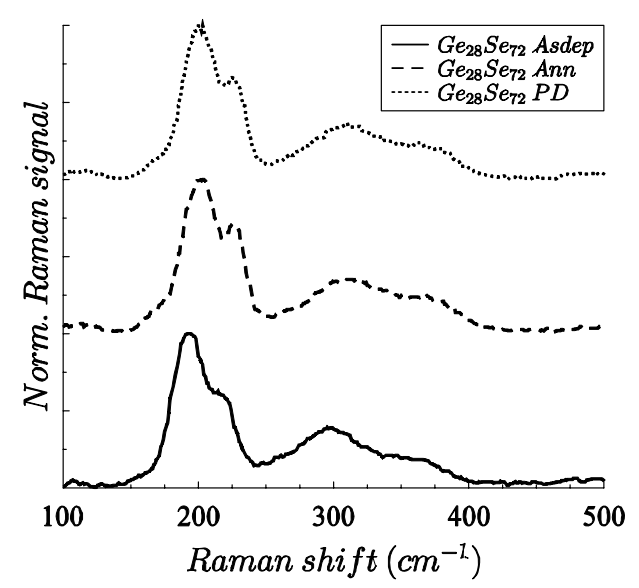

(1)

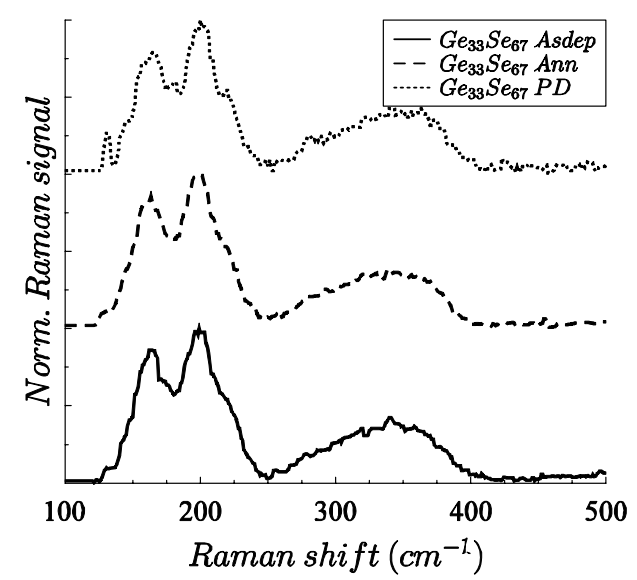

(2)

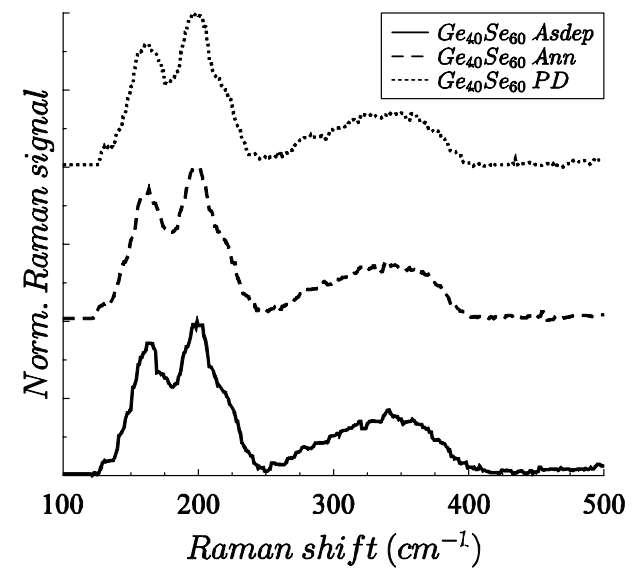

(3)

Fig. 5 Raman spectra of the as-deposited, annealed and irradiated after annealing Ge-Se (PD state) samples, (1) $\mathrm{Ge}_{28} \mathrm{Se}_{72},(2)-\mathrm{Ge}_{33} \mathrm{Se}_{67}$ and (3) $-\mathrm{Ge}_{40} \mathrm{Se}_{60}$.

corner sharing structural units. This leads to modification of the structure and formation of new edge sharing units, as is was revealed by the Raman spectroscopy (Fig. 3). So the PD probably has the same origin as in the As-based chalcogenides and can be explained by bond breaking, switching and atomic movement in the structure [26]. However, we would like to emphasise the importance of the electron excitation of the lone pair electrons, which we assume play an important role in the weakening of the bonds and their subsequent breaking. The freshly evaporated films have a random structure, with a lot of Se-Se and Ge-Ge bonds. Due to laser irradiation, the structure shifts to equilibrium state, characterized by increased number of heteropolar bonds, which are strongly preferred. The PD can be connected with this bond rearrangement, which results in a red shift of the absorption edge and increase of the absorption coefficient. This contributes to the opening of the structure and, as a consequence, to the increase in the films thickness, as it was revealed by our measurements and also reported for some Ge-As-Se glasses [27].

The modification of the structure also affects the optical band gap and the refractive index of the films. The relationship between these changes has already been reported for similar materials [28]. The decreasing optical band gap is related to the reorganization of the electronic atomic structure after the electron-hole pair is formed due to illumination. .

After the complete saturation of the PD process, the PB starts to develop and it saturates with time. A close look at the graph presented in Fig. 2 suggests that the $\mathrm{PB}$ effect is much slower than the PD. One of the reasons of the $\mathrm{PB}$ effect can be further homopolar bond breaking, occurring in a quite limited fashion, compared to the homopolar bonds reduction related to the PD process, similarly to the results reported in $[22,24]$. The ratio of Raman peak areas of the building blocks is quite close to that observed for relaxed bulk material with the same composition [29]. We suggest that during $\mathrm{PB}$, the films in fact approach their equilibrium condition. The increase of the ES peak area would mean that the structure is compacting, in contrary 
Table 4 Area of the major peaks in the Raman spectrum of the as-deposited, annealed and irradiated annealed Ge-Se samples, determined by fitting with Gaussians.

\begin{tabular}{llllllllll}
\hline & \multicolumn{3}{c}{$\mathrm{Ge}_{28} \mathrm{Se}_{72}$} & \multicolumn{3}{c}{$\mathrm{Ge}_{33} \mathrm{Se}_{67}$} & \multicolumn{3}{c}{$\mathrm{Ge}_{40} \mathrm{Se}_{60}$} \\
\cline { 2 - 10 } & Asdep & Ann & PD & Asdep & Ann & PD & Asdep & Ann & PD \\
\hline Se-Se & 10.6 & 10.3 & 10.1 & 19.1 & 18.6 & 17.8 & 15.4 & 15.3 & 15.2 \\
ES & 7.2 & 7.4 & 6.6 & 5.1 & 5.3 & 4.8 & 3.4 & 3.6 & 3.6 \\
CS & 22.3 & 21.9 & 22.5 & 19.1 & 18.8 & 19.3 & 13.0 & 13.4 & 13.2 \\
ETH & - & - & - & 4.3 & 4.0 & 3.9 & 23.8 & 23.1 & 22.7 \\
\hline
\end{tabular}

to its opening during the PD, and the thickness of the films reduces, as measured in this work. The second reason of the $\mathrm{PB}$ process could be the well known phenomenon of photo-oxidation in these materials, which can occur during illumination of Ge-based chalcogenide thin films in air $[13,15]$. Although there are some speculations that both elements $\mathrm{Ge}$ and $\mathrm{Se}$ could oxidize, following our experience [15], we believe that the only element that could form oxides in the system should be Ge. We have not observed vibrations of Ge-O bonds at $520-650 \mathrm{~cm}^{-1}$ [30], and believe that this process presumably should be excluded from the explanation of the studied phenomena.

The annealing contributes to structural and optical changes similar to those characteristics for the PB process, such as the blue shift of the optical band gap. Thus, the annealing process contributes towards relaxation of the structure and brings the films closer to their equilibrium state. In fact, annealing is often used to erase photo-darkening [6], and we suggest that it is also the reason of the absence of the PB in the annealed samples, since the relaxation has been established by annealing.

We would like to emphasise the compositional dependence of the effects which manifest a decreasing of the material's reaction towards light when the Ge content in the systems increases. We consider this as one more prove of the importance of the lone pair electrons for the occurring effects. Their formation and effect over the Ge-Se bonding makes the systems more sensitive towards light illumination. The decreased amount of chalcogen atoms in the Ge rich sample reduces the mass effect of these electrons, and combined with the rigidity of the material reduces the range of the illumination induced effects [31]. The results on photo-induced volume change and the additional possibility of mass transport in Ge-Se system under the gradient intensity of illumination show a potential of these materials for surface relief recording, as it was investigated and presented for As-Se and As-S layer structures [32, 33].

\section{Conclusions}

A fast decrease (photo-darkening) and a slow increase (photo-bleaching) of the optical transmission were observed during laser irradiation of $\mathrm{Ge}-\mathrm{Se}$ samples of different composition. The photo-darkening was found to be related to photo-induced bond switching with homopolar bond breaking and atomic rearrangement, similarly to As-based chalcogenide glasses. The PB can also be related to homopolar bond breaking occurring with simultaneous medium range ordering. We established a compositional dependence of the effects occurring emphasising the importance of the lone-pair electrons in the system. In relation to this, it was found from investigations of the studied samples, that $\mathrm{Ge}_{28} \mathrm{Se}_{27}$ is the composition with the best expressed photo-induced effects which diminish with increasing of Ge concentration in the samples.

\section{Acknowledgements}

This work was supported by TÁMOP 4.2.4. A/2-11-1-2012-0001 (Nemzeti Kiválóság Program) and TAMOP 4.2.2.A-11/1/KONV-2012-0036 projects, which are co-financed by the European Union and European Social Fund and by the Defense Threat Reduction Agency under grant no: HDTRA1-11-1-0055. The authors would like to thank 
Dr. James Reed of DTRA for his support.

\section{References}

[1] Popescu M. 2000. Non-Crystalline Chalcogenides. Solid State Science Technology Library: Kluwer Academic Publishers.

[2] Venger, E., Melnichuk, A., and Stronskyi, A. 2007. Photostimulated Processes in Vitreous Chalcogenide Semiconductors and Their Applications. Kiev: Academperiodica.

[3] Hisakuni, H., and Tanaka, K. 1995. "Optical Microfabrication of Chalcogenide Glasses." Science 270: 974-5.

[4] Shimakava, K., Yoshida, N., Ganjoo, A., Kuzukawa, Y., and Singh, J. 1998. "A Model for Photostructural Changes in Amorphous Chalcogenides." Phil. Mag. Letters 77: 889.

[5] Chen, G., Jain, H., Vlcek, M., and Ganjoo, A. 2006. "Photoinduced Volume Change in Arsenic Chalcogenides by Band-Gap Light.” Phys. Rev. B. 74: 10565.

[6] Tanaka, K., and Shimakawa, K. 2011. Amorphous Chalcogenide Semiconductors and Related Materials. New York: Springer.

[7] Trodahl, H. J., and Vina, L. 1983. "Visible-Near-uv Optical Spectra of a-Ge $\mathrm{Ge}_{1-\mathrm{x}}$." Phys. Rev. B. 27: 6498.

[8] Tichy, L., Ticha, H., Vlcek, M., Klikorka, J., and Jurek, K. 1986. "Photoinduced Bleaching of Amorphous Film $\mathrm{Ge}_{40} \mathrm{Se}_{60} . "$ J. Mater. Sci. Lett. 5: 1125-8.

[9] Broese, E., Schroter, B., Lehmann, A., Richter, A., and Schirmer, G. 1991. "Optical Properties of Quasi-Simultaneous Sputtered $\mathrm{Ge}_{\mathrm{x}} \mathrm{Se}_{1-\mathrm{x}}$. . J. Non-Cryst. Solids 130: 52-7.

[10] Kotkata, M., Kandil, K., And Theye, M. 1993. "Optical Studies of Disorder and Defects in Amorphous $\mathrm{Ge}_{\mathrm{x}} \mathrm{Se}_{1-\mathrm{x}}$ Films as a Function of Composition." J. Non-Cryst. Solids 164: 1259-1262.

[11] Petkov, K. 2002. "Compositional Dependence of the Photoinduced Phenomena in Chalcogenide Films." $J$. Optoelectron. Adv. Mater. 4: 611-629.

[12] Asatryan, K. E., Frederic, S., Galstian, T., and Vallee, R. 2004. "Recording of Polarization Holograms in Photodarkened Amorphous Chalcogenide Films." Appl. Phys. Lett. 84: 1626-8.

[13] Lyubin, V., Klebanov, M., Bruner, A., Shitrit, N., and Sfez, B. 2011. "Transient Photodarkening and Photobleaching in Glassy GeSe $\mathrm{C}_{2}$ Films.” Opt. Mater. 33: 949-952.

[14] Barik, A. R., Naik, R., and Adarsh, K. V. 2013. "Unusual Observation of Fast Photodarkening and Slow Photobleaching in a-GeSe2 Thin Film." J. Non.-Cryst. Solids 377: 179-181.

[15] Sakaguchi, Y., Tenne, D. A., and Mitkova, M. 2009.
"Oxygen-Assisted Photoinduced Structural Transformation in Amorphous Ge-S Films." Phys. Status Solidi B 246: 1813-9.

[16] Yan, Q., Jain, H., Ren, J., Zhao, D., and Chen, G. 2011. "Effect of Photo-Oxidation on Photobleaching of $\mathrm{GeSe}_{2}$ and $\mathrm{Ge}_{2} \mathrm{Se}_{3}$ Films." J. Phys. Chem. C 115: 21390-5.

[17] Spence, C. A., and Elliott, S. R. 1989. "Light-Induced Oxidation and Band-Edge Shifts in Thermally Evaporated Films of Germanium Chalcogenide Glasses." Phys. Rev. B Condens. Matter 39: 5452-5463.

[18] Yang, G., Gueguen, Y., Sangleboeuf, J.-C., Rouxel, T., Boussard-Plédel, C., Troles, J., and et al. 2013. "Physical Properties of the GexSe1-x Glasses in the $0<\mathrm{x}<0.42$ Range in Correlation with Their Structure." J. Non-Cryst. Sol. 377: 54-9.

[19] Swanepoel, R. 1983. "Determination of the Thickness and Optical Constants of Amorphous Silicon.” J. Phys. E-Sci. Instrum. 16: 1214-1222.

[20] Jackson, K., Briley, A., Grossman, S., Porezag, D. V., and Pederson, M. R. 1999. "Raman-Active Modes of a GeSe2 and a-GeS2: a First-Principles Study." Phys. Rev. $B$ 60: 14985-9.

[21] Shatnawi, M. T., Farrow, C. L., Chen, P., Boolchand, P., Sartbaeva, A., Thorpe, M., and et al. 2008. "Search for a Structural Response to the Intermediate Phase in $\mathrm{Ge}_{\mathrm{x}} \mathrm{Se}_{1-\mathrm{x}}$ Glasses." Phys. Rev. B 77: 094134.

[22] Vateva, E., and Arsova, D. 2010. "Transition of Reversible Photodarkening to Photobleaching in Chalcogenide Films." EPL 89: 64004-8.

[23] Arsova, D., and Vateva, E. 2012. "Dual Action of Light in Photodarkened Ge-As-S Films." Phys. Stat. Sol. B 249: 153-7.

[24] Khan, P., Jain, H., and Adarsh, K. V. 2014. "Role of Ge:As Ratio in Controlling the Light-Induced Response of a- $\mathrm{Ge}_{\mathrm{x}} \mathrm{As}_{35-\mathrm{x}} \mathrm{Se}_{65}$ Thin Films." Sci. Reports 4: 4029.

[25] Elliott, S. R. 1986. "A Unified Model for Reversible Photostructural Effects in Chalcogenide Glasses." $J$. Non-Cryst. Sol. 81: 71-98.

[26] Shimakawa, K., Kolobov, A., and Elliott, S. R. 1995. "Photoinduced Effects and Metastability in Amorphous Semiconductors and Insulators." Adv. Phys. 44: 475-588.

[27] Edwards, T. G., and Sen, S. 2011. "Structure and Relaxation in Germanium Selenide Glasses and Supercooled Liquids: A Raman Spectroscopic Study." J. of Phys. Chem. B 115: 4307-4314.

[28] Orava, J., Kohoutek, T., Wagner, T., Cerna, Z., Vlcek, M., Benes, L., and et al. 2009. "Optical and Structural Properties of Ge-Se Bulk Glasses and Ag-Ge-Se Thin Films.” J. Non-Cryst. Sol. 355: 1951-4.

[29] Mitkova, M., Wang, Y., and Boolchand, P. 1999. "Dual Chemical Role of $\mathrm{Ag}$ as an Additive in Chalcogenide Glasses." Phys. Rev. Lett. 83: 3848-3851. 
[30] Kim, Y., Saienga, J., and Martin, S. W. 2005. "Preparation and Characterization of Germanium Oxy-Sulfide GeS2-GeO2 Glasses." J. Non-Cryst. Sol. 351: 1973-9.

[31] Calvez, L., Yang, Z., and Lucas, P. 2008. "Light-Induced Matrix Softening of Ge-As-Se Network Glasses." Phys. Rev. Lett. 101: 2411-5.
[32] Teteris, J., Gertners, U., and Reinfelde, M. 2011. "Photoinduced Mass Transfer in Amorphous $\mathrm{As}_{2} \mathrm{~S}_{3}$ Films." Phys. Stat. Solidi C 8: 2780-4.

[33] Trunov, M., Lytvyn, P., Takats, V., Charnovich, I., and Kokenyesi, S. 2009. "Direct Surface Relief Formation in As0.2Se0.8 Layers." J. Optoelectron. Adv. Mater. 11: 1959-1962. 